\title{
Nonspatial Attentional Shifts Between Audition and Vision
}

\author{
Massimo Turatto, Francesco Benso, Giovanni Galfano, and Carlo Umiltà \\ University of Padua
}

\begin{abstract}
This study investigated nonspatial shifts of attention between visual and auditory modalities. The authors provide evidence that the modality of a stimulus $\left(S_{1}\right)$ affected the processing of a subsequent stimulus $\left(S_{2}\right)$ depending on whether they shared the same modality. For both vision and audition, the onset of $S_{1}$ summoned attention exogenously to its modality, causing a delay in processing $\mathrm{S}_{2}$ in a different modality. That undermines the notion that auditory stimuli have a stronger and more automatic alerting effect than visual stimuli (M. I. Posner, M. J. Nissen, \& R. M. Klein, 1976). The results are consistent with other recent studies showing cross-modal attentional limitation. The authors suggest that such cross-modal limitation can be produced by simply presenting $S_{1}$ and $S_{2}$ in different modalities and that central processing mechanisms are also, at least partially, modality dependent.
\end{abstract}

In everyday life, people commonly interact with a multisensorial environment, in which attention becomes relevant for the selection and coordination of stimuli coming from different sensory modalities. Hence, of particular interest is the study of how selective attention gates the flow of information processing in situation in which stimulation of different sensory systems is provided concurrently or in close temporal proximity. In this regard, there is evidence of interference in processing stimuli coming simultaneously from different modalities (e.g., Egeth \& Sager, 1977; Shiffrin \& Grantham, 1974). Cross-modal interference or facilitation has been shown to occur even when two stimuli are delivered separated by a temporal interval. Early studies showed that when two stimuli are presented one after the other, the modality of the first stimulus $\left(\mathrm{S}_{1}\right)$ affects the processing of the second stimulus $\left(\mathrm{S}_{2}\right)$, although $\mathrm{S}_{1}$ modality provides no information about $\mathrm{S}_{2}$ modality (Harvey, 1980; Posner, Nissen, \& Klein, 1976). In fact, one might expect cross-modal conditions to produce great interference, because the limited capacity has to be serially deployed to each modality in turn (see Boulter, 1977; Moore \& Massaro, 1973). In contrast, what emerged from Posner et al.'s (1976; also see Harvey, 1980) study was that response times (RTs) to a visual $\mathrm{S}_{2}$ were shorter after an auditory $\mathrm{S}_{1}$ than after a visual $\mathrm{S}_{1}$.

According to Posner et al. (1976; also see Klein, 1977), this advantage for auditory $S_{1}$ occurs because visual signals are less alerting than stimuli in other modalities. In particular, it was suggested that auditory and kinesthetic stimuli have an automatic

Massimo Turatto, Francesco Benso, and Carlo Umiltà, Department of General Psychology, University of Padua, Padua, Italy; Giovanni Galfano, Department of Developmental Psychology, University of Padua, Padua, Italy.

The research was supported by grants from Consiglio Nazionale delle Ricerche (CNR) and Ministero dell'Università e della Ricerca Scientifica e Tecnologica (MURST) to Carlo Umiltà. We are grateful to Charles Spence, Bruce Bridgeman, and two anonymous reviewers for their many constructive criticisms of an earlier version of this article. Thanks are also due to Andrea Pierno for testing participants in Experiments 5 and 6.

Correspondence concerning this article should be addressed to Carlo Umiltà, Dipartimento di Psicologia Generale, Università degli Studi di Padova, Via Venezia 8, 35131 Padova, Italy. E-mail: carlo.umilta@ unipd.it alerting effect and are able to activate the central decision-making mechanisms without the intervention of attention. By contrast, the effortful alerting effect of visual stimuli heavily depends on attention being directed to them. Automatic alertness would have a nonspecific effect of activation over the cognitive system, making it ready to respond to every kind of stimulus, regardless of its modality. In contrast, the effortful alertness would be modality specific.

The hypothesized low alertness of visual stimuli would be one of the causes that produce the visual dominance phenomenon, namely, that observers' performance in different multisensorial tasks tends to be controlled by visual information (e.g., Colavita, 1974; Gibson, 1943). The phenomenon manifests itself in various tasks, such as perceptual and memory judgments, motor performance, and RTs (for a detailed review, see Posner et al., 1976).

More recently, the relation between attention and sensory modalities has been investigated from a different perspective, which was more concerned with the role of spatial attention and the cross-modal representation of space (Driver \& Spence, 1994, 1998). In particular, the demonstration of such cross-modal links in spatial attention was based on the observation that a noninformative spatial cue in one modality would capture covert attention toward its position in other modalities (e.g., Spence \& Driver, 1997a).

For the purpose of the present study, the work of Spence and Driver $(1994,1996,1997 a, 1997 b)$ is of relevance for another reason. Spence and Driver pointed out a series of methodological artifacts that were often present in previous studies, leaving alternative nonattentional accounts viable (for a detailed discussion, see Spence \& Driver, 1997b). The first nonattentional account identified by Spence and Driver is the response priming artifact. Basically, this artifact emerges in experimental designs that compare RTs in modality-certain blocks with RTs in modality-uncertain blocks (e.g., Verleger \& Cohen, 1978). Instead of being ascribed to attention, advantage for the blocks in which target modality is certain may simply be ascribed to participants knowing the appropriate response before target presentation. This leads to an erroneous comparison between a simple RT task (modality-certain blocks) and a choice RT task (modality-uncertain blocks). 
A further common methodological flaw is the criterion shift, which reflects the tendency of participants to lower their criterion for responding to events in the expected modality (e.g., Kristofferson, 1967). This usually happens when participants perform a simple RT task in which target modality is certain. A criterion shift may be identified because the decreased RTs observed for the certain modality may be accompanied by an increased number of false alarms (i.e., responses to catch trials).

Another confounding factor is extremely important, namely, the spatial artifact. This occurs when the sources of stimulation for different sensory modalities are not spatially coincident, with the resulting attentional costs likely due to a spatial movement of attention rather than to a real shift of attention across modalities. This artifact was often present in previous studies, in which visual stimuli were shown on a visual display located in front of the participant, whereas auditory stimuli were delivered through headphones (e.g., Harvey, 1980; Klein, 1977; Posner et al., 1976).

Spence and Driver (1997b) highlighted yet another common methodological flaw that could have affected previous experimental findings, namely, the modality shift effect (MSE). It refers to the possibility that "regardless of any expectancy, there may be some cost in responding to targets when their modality changes successively, or some benefit in responding to a repeated sequence of identical stimuli" (Spence \& Driver, 1997b, p. 390). The MSE is a purely exogenous effect that can be described as the automatic deployment of attention to the stimulated modality. It should be clear that, if one is interested in exploring the possible spatial attention links across expected modalities, the MSE might be a confounding factor. However, for our purposes, the MSE, or exogenous attentional capture, as we prefer to label it, is not an artifact but rather one of the central issues with which the present study is concerned.

\section{Aims of the Research}

Apart from early studies (e.g., Boulter, 1977; Harvey, 1980; Klein, 1977; Posner et al., 1976), the issue of nonspatial shifts of attention across modalities has been somewhat neglected in the recent literature on cross-modal attention. The goal of the present research was to readdress nonspatial shifts of attention between audition and vision, originally studied in the 1970s, by avoiding the methodological flaws highlighted earlier.

We used a unique spatial source of stimulation for visual and auditory events to investigate exogenous and endogenous allocation of attention between modalities. In particular, in accordance to what is predicted by the MSE, we hypothesized that when $S_{1}$ is presented in a given modality, attention is automatically summoned to that same modality. Therefore, if a target $S_{2}$ is briefly presented, RTs to the target should be shorter for modalitycongruent trials than for modality-incongruent trials. Contrary to this prediction, previous studies seemed to provide evidence for a greater facilitatory effect of an auditory than a visual $S_{1}$ on a visual $S_{2}$ (e.g., Posner et al., 1976).

Also, it seems quite reasonable to assume that, if $S_{1}$ modality is used as a predictor of $S_{2}$ modality, the observer might endogenously direct attention to the cued modality. In addition, if attention is automatically grabbed to $S_{1}$ modality even when $S_{1}$ is noninformative of $S_{2}$ modality, one might expect attention to be voluntarily directed more efficiently to $S_{2}$ modality in ipsimodal trials than in cross-modal trials.
Experimental conditions were planned taking into consideration the methodological criteria proposed by Spence and Driver (1997b). Experiments 1 through 4 were based on a simple RT task. On each trial, participants were delivered an auditory or visual $S_{1}$, which provided, in a probabilistic manner (Experiment 1, 50\%; Experiments 2 and 3, 80\%; Experiment 4, 100\%), information about $\mathrm{S}_{2}$ (the target) modality. By using a trial-by-trial procedure, we eliminated any response priming artifacts. Catch trials were also presented to discourage participants from anticipating their response, thus lessening the problem of a criterion shift in simple RT tasks. Experiments 5 and 6 required a discrimination task and were meant to definitively rule out any criterion shifts.

Because in the present study we deal with stimuli from different sensory channels, phenomenological and qualitative differences between modalities are no doubt present. However, trying to equate modalities for intensity, localizability, or alerting effectiveness would likely not be sufficient to render equivalent the stimuli for the two different modalities. There is no way to ensure that the two stimuli are equivalent. Therefore, we used visual and auditory stimuli of moderate intensities, which we judged as to be roughly equivalent. Note that, to our knowledge, none of the previous studies made any particular efforts to equate perceptually auditory and visual stimuli (e.g., Spence \& Driver, 1994, 1996, 1997a, 1997b). However, it is worth noting that Experiments 5 and 6, in which criterion shifts were eliminated, indicated that, for the longest $S_{1}-S_{2}$ interval $\left(1,000 \mathrm{~ms}\right.$; i.e., when $S_{2}$ is least affected by $\mathrm{S}_{1}$ modality), RTs on visual and auditory ipsimodal trials did not differ.

\section{Experiment 1}

In this experiment we investigated whether attention is automatically captured by the modality of $S_{1}$ when participants are requested to process a subsequent $S_{2}$ and both stimuli are delivered from the same location. ${ }^{1}$ If this were the case, we expected $S_{1}$ to produce RT benefits in processing a subsequent ipsimodal $S_{2}$ in comparison with a cross-modal $S_{2}$. Because $S_{1}$ modality was not predictive of $S_{2}$ modality, participants had no incentive to use any deliberate strategy to allocate attention to $S_{1}$ modality, and thus the role of expectancy-endogenous factors should be minimized, if not totally excluded. Three stimulus onset asynchronies (SOAs) between $S_{1}$ and $S_{2}$ were used.

\section{Method}

Participants. Twenty-five psychology students from the University of Padua, Padua, Italy, served as participants (16 female and 9 male). All were unaware of the purpose of the experiment. Their mean age was 26 years (range $=20-29$ years). All were right-handed by self-report and reported normal hearing and normal or corrected-to-normal vision.

Apparatus and materials. The present experiment and all the following ones were conducted in a dim-lit room with a background luminance of

\footnotetext{
${ }^{1}$ Although we used a device in which the centers of stimulation were at the same position in azimuth, still the auditory and visual modalities might not be perfectly coincident in space. Thus, one cannot be certain that the modalities were perceptually coincident either. However, the experimental setting we used was such as to limit the possibility of a spatial shift, because the spatial source was the same for both modalities. That presumably allowed participants to have a clear location to attend to, regardless of what modality was actually stimulated.
} 
about $0.5 \mathrm{~cd} / \mathrm{m}^{2}$. The participant sat at a table facing an audiovisual device composed of a box in which a circle loudspeaker cone $(3 \mathrm{~cm}, 8 \Omega, 200$ $\mathrm{mW}$, RS 248-476) and a light-emitting diode (LED; $1.15^{\circ}$ of visual angle) were assembled coaxially: The loudspeaker was mounted just behind the LED (see Figure 1).

For the visual modality, $\mathrm{S}_{1}$ was the onset of the LED in a red color (CIE coordinates, $x=.675, y=.324 ; 140 \mathrm{~cd} / \mathrm{m}^{2}$ ), whereas $\mathrm{S}_{2}$ was the onset of the same LED in green color (CIE coordinates, $x=.424, y=.572 ; 232$ $\left.\mathrm{cd} / \mathrm{m}^{2}\right)$. For the auditory modality, $\mathrm{S}_{1}$ was a $900-\mathrm{Hz}$ pure tone presented at $70 \mathrm{~dB}(\mathrm{~A})$, whereas $\mathrm{S}_{2}$ was a $1800-\mathrm{Hz}$ pure tone presented at $70 \mathrm{~dB}(\mathrm{~A})$. Stimuli were provided by a 486 personal computer, and RTs were measured in milliseconds. Timing of the stimuli and responses was ensured by using a program written in Micro Experimental Laboratory (Schneider, 1988), which ensures 1-ms time precision.

Design and procedure. Before the experiment began, participants were clearly informed that $S_{1}$ modality was not predictive of $S_{2}$ modality. They performed practice trials until they felt confident with the task. Then there were three experimental blocks of 36 trials each (not counting catch trials). Specifically, there were 12 trials for each SOA, 6 trials for each congruent modality $\mathrm{S}_{1}-\mathrm{S}_{2}$ combination, and 6 for each incongruent modality $\mathrm{S}_{1}-\mathrm{S}_{2}$ combination $(25 \%$ visual-visual, $25 \%$ visual-auditory, $25 \%$ auditoryauditory, and $25 \%$ auditory-visual). Catch trials were 8 per block (about $18 \%$ of total trials). On catch trials, participants had to refrain from responding.

The beginning of a new trial was signaled by the simultaneous onset of the LED in orange color (CIE coordinates, $x=.538, y=.416 ; 385 \mathrm{~cd} / \mathrm{m}^{2}$ ), along with the $900+1800-\mathrm{Hz}$ tone, presented together at $70 \mathrm{~dB}(\mathrm{~A})$ for $1,000 \mathrm{~ms}$. Then the LED and the tone were turned off, and 1,000 ms later $\mathrm{S}_{1}$ was presented for $100 \mathrm{~ms}$. After a variable SOA $(150,600$, and 1,000 $\mathrm{ms}), \mathrm{S}_{2}$ (the target) was turned on for $100 \mathrm{~ms}$. Participants were required to press the space bar of the computer keyboard as soon as the target was detected and to refrain from responding on catch trials, in which $\mathrm{S}_{1}$ was not followed by $\mathrm{S}_{2}$. Allowed time for responding was $1,000 \mathrm{~ms}$.

\section{Results}

Overall false alarms (i.e., responses on catch trials) were very low, 1 out of 300 total responses for auditory modality and 1 out of 300 for visual modality, and were not further analyzed. In this and the following experiments, trials on which RTs were shorter than $150 \mathrm{~ms}$ or longer than 2.5 standard deviations from the mean were treated as outliers and were eliminated prior to formal data analysis. In the present experiment, this latency criterion removed less than $3 \%$ of data. The following conventions are used: VV (visual $S_{1}$ followed by visual $S_{2}$ ), AV (auditory $S_{1}$ followed by visual $S_{2}$ ), AA (auditory $S_{1}$ followed by auditory $S_{2}$ ), and VA (visual $S_{1}$ followed by auditory $S_{2}$ ).

RTs were entered into a repeated measures analysis of variance (ANOVA), in which the factors were $S_{1}$ modality, $S_{2}$ modality,

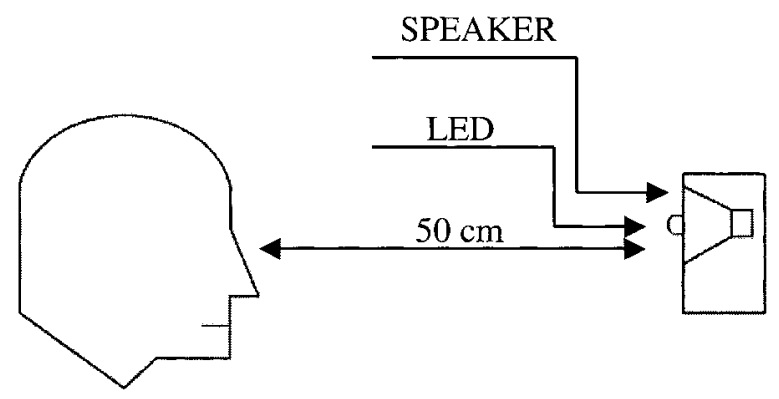

Figure 1. Schematic drawing of the apparatus used in every experiment. LED $=$ light-emitting diode. and SOA. The main effect of $S_{1}$ modality was not significant, whereas the main effect of $\mathrm{S}_{2}$ modality was significant, $F(1,24)=$ 26.946, $p<.001$, with participants responding more rapidly to the auditory target $(M=411 \mathrm{~ms}, S D=92)$ than to the visual target $(M=444 \mathrm{~ms}, S D=87)$. There was also a significant main effect of SOA, $F(2,48)=54.827, p<.001$, with participants responding more rapidly at the intermediate SOA (150-ms SOA, $M=469 \mathrm{~ms}$, $S D=99 ; 600-\mathrm{ms}$ SOA, $M=395 \mathrm{~ms}, S D=72 ; 1,000-\mathrm{ms}$ SOA, $M=419 \mathrm{~ms}, S D=84)$. This likely was a regular foreperiod effect, with RTs becoming shorter as SOA increases (e.g., Bertelson, 1967). It was present in every experiment and is not discussed further. The $\mathrm{S}_{1}$ Modality $\times \mathrm{S}_{2}$ Modality interaction was significant, $F(1,24)=13.680, p<.005(\mathrm{VV}, M=437 \mathrm{~ms}, S D=85$; $\mathrm{AV}, M=451 \mathrm{~ms}, S D=90$; $\mathrm{AA}, M=395 \mathrm{~ms}, S D=83$; VA, $M=$ $427 \mathrm{~ms}, S D=98)$, with participants responding more rapidly when $\mathrm{S}_{1}$ modality and $\mathrm{S}_{2}$ modality were congruent $(M=416 \mathrm{~ms}$, $S D=81)$ than when they were incongruent $(M=439 \mathrm{~ms}, S D=$ $88)$. The $S_{1}$ Modality $\times S_{2}$ Modality $\times$ SOA interaction was also significant, $F(2,48)=60.402, p<.001$, showing that the advantage of congruent-modality trials varied as a function of SOA. As shown in Figure 2 and Table 1, RTs on congruent-modality trials were significantly shorter than RTs on incongruent-modality trials only at the shortest SOA (150 ms).

Planned comparisons were applied to the data. At the shortest SOA (150 ms), RTs in VV were shorter than in AV (mean difference $=74 \mathrm{~ms}, p<.01)$; similarly, RTs in AA were shorter than in VA (mean difference $=101 \mathrm{~ms}, p<.01$ ). By contrast, at the 600-ms SOA, RTs in VV and AV did not significantly differ, nor did those in AA and VA. At the longest SOA (1,000 ms), participants were faster in $\mathrm{AV}$ than in $\mathrm{VV}$ (mean difference $=27$ $\mathrm{ms}, p<.01$ ) and in VA than in AA (mean difference $=21 \mathrm{~ms}, p<$ $.05)$.

\section{Discussion}

Our main prediction was straightforward: The mere presentation of a stimulus $\left(\mathrm{S}_{1}\right)$ in a given modality produces an exogenous allocation of attention to that modality. Hence, when a second stimulus $\left(\mathrm{S}_{2}\right)$ is presented, its detection is faster when the two stimuli share the same modality than when they are shown in different modalities.

At the shortest SOA $(150 \mathrm{~ms})$, participants were faster at detecting $\mathrm{S}_{2}$ on ipsimodal trials than on cross-modal trials. In accord with our hypothesis, this result is explained by assuming that the onset of $\mathrm{S}_{1}$ summoned attention to the stimulated modality. On congruent trials, RT for $\mathrm{S}_{2}$ detection benefits when attention is already allocated to the correct modality. In contrast, RT on cross-modal trials is lengthened because attention must be shifted to $S_{2}$ modality before the response can be executed.

Because $S_{1}$ and $S_{2}$ modalities were completely uncorrelated, and because the task was very simple, participants did not have any incentive to attend to a specific modality. Therefore, it seems reasonable to assume that the effects observed were not due to an endogenous allocation of attention to $S_{1}$ modality.

In our view, because stimuli originated from the same spatial position, the shorter RT observed for ipsimodal trials cannot be explained either by postulating the intervention of a spatial orient- 
ing process. $^{2}$ Rather, the results reflect a nonspatial attentional effect, purely attributable to the automatic allocation of attention to $\mathrm{S}_{1}$ modality.

The advantage of ipsimodal trials over cross-modal trials disappeared at the intermediate SOA $(600 \mathrm{~ms})$. Probably, participants, knowing that $S_{1}$ and $S_{2}$ modalities were uncorrelated, successfully counteracted the automatic tendency to allocate attention to $S_{1}$ modality. An SOA of $600 \mathrm{~ms}$ was long enough to adopt this voluntary strategy, whereas an SOA of $150 \mathrm{~ms}$ was too short.

At the longest SOA $(1,000 \mathrm{~ms})$, the facilitatory effect present at the 150-ms SOA reversed. At first glance, this inhibitory effect might resemble what, in the spatial attention domain, is called inhibition of return (IOR; e.g., Posner \& Cohen, 1984; see Klein, 2000 , for a recent review). The IOR phenomenon consists in a bias against returning spatial attention to a recently explored location. However, on the basis of the present evidence, we cannot conclude that a nonspatial IOR-like mechanism operated when the $S_{1}-S_{2}$ interval was $1,000 \mathrm{~ms}$. For the time being, we can only suppose that the effect is due to attention. Future work is needed to ascertain whether that effect is a new kind of IOR and whether it has characteristics and a time course similar to spatial IOR.

Early studies had shown an advantage for AV over VV (e.g., Harvey, 1980; Nickerson, 1973; Posner et al., 1976; Sanders, 1975). In contrast, we found that RTs in VV were shorter than in $A V$, and that an auditory $S_{1}$ delayed RTs to a visual $S_{2}$ as much as a visual $S_{1}$ delayed RTs to an auditory $S_{2}$. This result undermines the distinction between an automatic alerting effect (typical of auditory stimuli) and an effortful alerting effect (typical of visual stimuli). Also, it would seem that two of the main conclusions of previous studies (Klein, 1977; Posner et al., 1976) should be considered with caution. The first is the idea that auditory stimuli would activate the central decision mechanism(s) without the intervention of attention. The second is that visual stimuli have a poorer alerting capability than auditory stimuli.

\section{Experiment 2}

Experiment 1 revealed that the sudden onset of a stimulus in a given modality produces an exogenous attentional capture to that

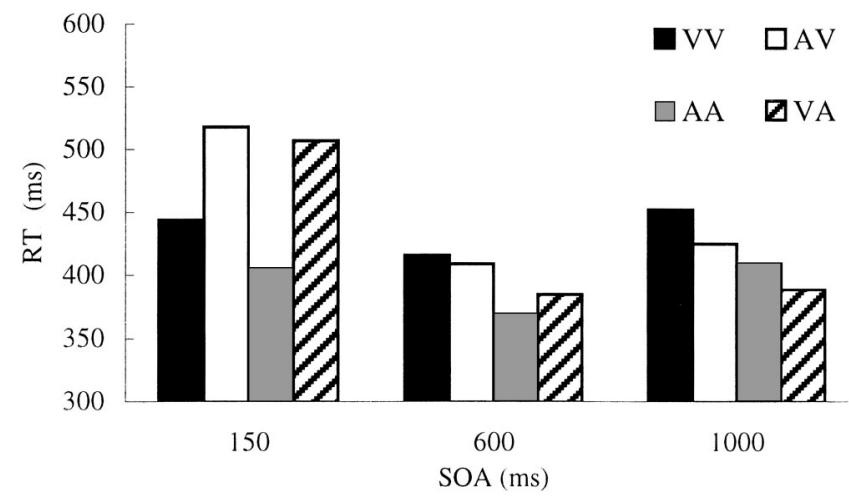

Figure 2. The $\mathrm{S}_{1}$ Modality $\times \mathrm{S}_{2}$ Modality $\times$ Stimulus Onset Asynchrony (SOA) interaction in Experiment $1 . \mathrm{S}_{1}$ (the first stimulus) modality was not informative about $\mathrm{S}_{2}$ (subsequent stimulus) modality. $\mathrm{RT}$ = response time; $\mathrm{VV}=$ visual $\mathrm{S}_{1}$ followed by a visual $\mathrm{S}_{2} ; \mathrm{AV}=$ auditory $\mathrm{S}_{1}$ followed by a visual $\mathrm{S}_{2}$; $\mathrm{AA}=$ auditory $\mathrm{S}_{1}$ followed by an auditory $\mathrm{S}_{2}$; VA $=$ visual $\mathrm{S}_{1}$ followed by an auditory $S_{2}$.
Table 1

Mean Reaction Times (in Milliseconds) and Standard

Deviations for Congruent and Incongruent Trials

in Experiments 1, 2, and 3

\begin{tabular}{|c|c|c|c|c|}
\hline \multirow[b]{3}{*}{ Experiment and SOA (ms) } & \multicolumn{4}{|c|}{ Trial congruence } \\
\hline & \multicolumn{2}{|c|}{ Congruent } & \multicolumn{2}{|c|}{ Incongruent } \\
\hline & $M$ & $S D$ & $M$ & $S D$ \\
\hline \multicolumn{5}{|l|}{ Experiment 1} \\
\hline 150 & 425 & 84 & 512 & 84 \\
\hline 600 & 392 & 70 & 396 & 59 \\
\hline 1,000 & 430 & 85 & 407 & 69 \\
\hline \multicolumn{5}{|l|}{ Experiment 2} \\
\hline 150 & $405^{\mathrm{a}}$ & 65 & $577^{\mathrm{b}}$ & 93 \\
\hline 600 & $353^{\mathrm{a}}$ & 56 & $455^{\mathrm{b}}$ & 67 \\
\hline 1,000 & $403^{\mathrm{a}}$ & 76 & $457^{\mathrm{b}}$ & 63 \\
\hline \multicolumn{5}{|l|}{ Experiment 3} \\
\hline 150 & $458^{\mathrm{b}}$ & 84 & $467^{\mathrm{a}}$ & 78 \\
\hline 600 & $436^{\mathrm{b}}$ & 67 & $367^{\mathrm{a}}$ & 74 \\
\hline 1,000 & $482^{\mathrm{b}}$ & 84 & $392^{\mathrm{a}}$ & 83 \\
\hline
\end{tabular}

Note. $\quad$ SOA $=$ stimulus onset asynchrony.

${ }^{\mathrm{a}}$ Also referred to as valid trials. ${ }^{\mathrm{b}}$ Also referred to as invalid trials.

modality. By using the same paradigm as in Experiment 1, we examined in Experiment 2 whether participants can voluntarily shift attention to a sensory modality by using an informative $(80 \%$ valid) congruent $S_{1}$. In spatial attention, two independent mechanisms for shifting attention are known to exist, one is stimulus driven or automatic and the other is goal directed or voluntary (e.g., Umiltà, 2000b). A possibility is that two similar mechanisms exist for shifting attention between modalities. Hence, Experiment 2 investigated this possibility.

\section{Method}

Participants. Twenty-four psychology students of the University of Padua (10 female and 14 male) participated. Their mean age was 25 years (range $=20-27$ years). All were right-handed by self-report and reported normal hearing and normal or corrected-to-normal vision. They were unaware of the purpose of the experiment and had not taken part in Experiment 1.

Apparatus and materials. The apparatus and materials were as in Experiment 1.

Design and procedure. The design and procedure were as in Experiment 1 , with the exception that $S_{1}$ validity was $80 \%$ for the congruent modality ( $80 \% \mathrm{VV}, 20 \% \mathrm{AV}, 80 \% \mathrm{AA}$, and $20 \% \mathrm{VA})$. There were three experimental blocks of 60 trials each (plus catch trials). There were 20 trials for each SOA, 16 trials for the congruent modality $\mathrm{S}_{1}-\mathrm{S}_{2}$ combination ( $8 \mathrm{VV}$ and $8 \mathrm{AA}$ ), and 4 trials for the incongruent modality $\mathrm{S}_{1}-\mathrm{S}_{2}$ combination ( $2 \mathrm{AV}$ and $2 \mathrm{VA}$ ). Catch trials were 12 in each block (about $17 \%$ of total trials).

\footnotetext{
${ }^{2}$ By stating that no spatial attention shifts are involved in the present study, we do not mean that spatial attention is not spatially allocated. No doubt, attention is voluntarily deployed to the spatial source of stimulation. In addition, the onset of $S_{1}$ might well grab attention automatically to its position. However, what is crucial is that, unlike what happens in crossmodal spatial attention studies, in which the location of the cue $\left(S_{1}\right)$ is varied as a function of the location of the target $\left(\mathrm{S}_{2}\right)$, in our study no shift of attention is required between $S_{1}$ and $S_{2}$.
} 


\section{Results}

Overall false alarms (i.e., responses on catch trials) were very few, 1 out of 432 total responses for the auditory $S_{1}$ and 0 out of 432 for the visual $S_{1}$, and were not further analyzed. As a result of the outliers-latency criterion, less than $1 \%$ of trials were trimmed. RT data were entered into a repeated measures ANOVA in which the factors were those already reported in Experiment 1. Both the main effect of $\mathrm{S}_{1}$ modality, $F(1,23)=7.488, p<.05$, and the main effect of $\mathrm{S}_{2}$ modality, $F(1,23)=49.131, p<.001$, were significant. RTs were shorter for auditory $\mathrm{S}_{1}(M=436 \mathrm{~ms}, S D=$ $109)$ than for visual $S_{1}(M=448 \mathrm{~ms}, S D=101)$, and shorter for auditory $\mathrm{S}_{2}(M=427 \mathrm{~ms}, S D=112)$ than for visual $\mathrm{S}_{2}(M=457$ $\mathrm{ms}, S D=96)$. The main effect of SOA was significant, $F(2,46)=$ $88.385, p<.001$, with shorter RTs at the two longer SOAs (150-ms SOA, $M=492 \mathrm{~ms}, S D=125 ; 600$-ms SOA, $M=404$ $\mathrm{ms}, S D=85 ; 1,000-\mathrm{ms}$ SOA, $M=430 \mathrm{~ms}, S D=80)$. A significant $\mathrm{S}_{1}$ Modality $\times \mathrm{S}_{2}$ Modality interaction was found, $F(1$, $23)=175.774, p<.0001(\mathrm{VV}, M=408 \mathrm{~ms}, S D=71$; $\mathrm{AV}, M=$ $507 \mathrm{~ms}, S D=93$; AA, $M=366 \mathrm{~ms}, S D=73$; VA, $M=488 \mathrm{~ms}$, $S D=111)$, with shorter RTs on valid (congruent) trials $(M=387$ $\mathrm{ms}, S D=70)$ than on invalid (incongruent) trials $(M=496 \mathrm{~ms}$, $S D=94)$. The $S_{1}$ Modality $\times S_{2}$ Modality $\times$ SOA interaction was also significant, $F(2,46)=37.874, p<.001$, showing that the difference between valid trials and invalid trials varied as a function of SOA (see Figure 3 and Table 1).

Planned comparisons were applied to the data, showing that, at each SOA, RTs in VV were shorter than in AV (150-ms SOA, mean difference $=136 \mathrm{~ms} ; 600-\mathrm{ms}$ SOA, mean difference $=105$ $\mathrm{ms}$; 1,000-ms SOA, mean difference $=56 \mathrm{~ms}$; all $p \mathrm{~s}<.01)$, and that RTs in AA were shorter than in VA (150-ms SOA, mean difference $=212 \mathrm{~ms}$; 600-ms SOA, mean difference $=99 \mathrm{~ms}$; 1,000 -ms SOA, mean difference $=55 \mathrm{~ms}$; all $p \mathrm{~s}<.01$ ).

Because of the voluntary component presumably involved in the present experiment, we expected greater differences than in Experiment 1 between valid-congruent and invalid-incongruent trials. Thus, because the advantage for ipsimodal trials was present in Experiment 1 at the shortest SOA only $(150 \mathrm{~ms})$, we compared the two experiments at this SOA. The between-participants factor was

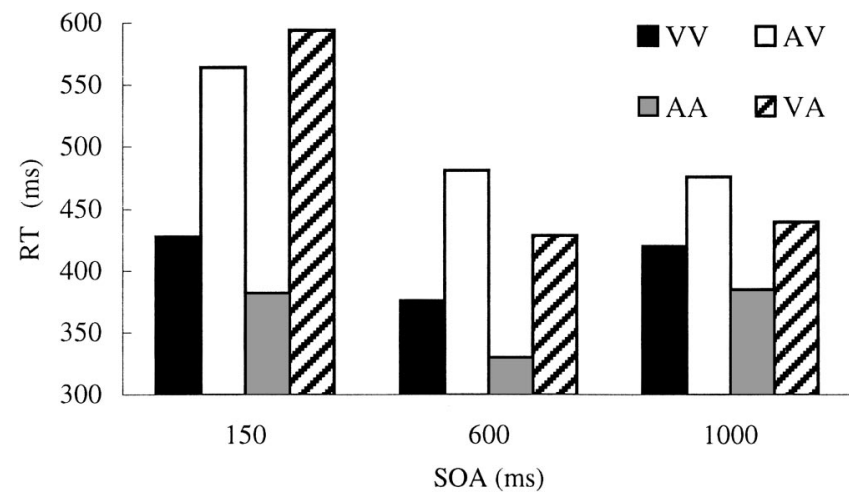

Figure 3. The $\mathrm{S}_{1}$ Modality $\times \mathrm{S}_{2}$ Modality $\times$ Stimulus Onset Asynchrony (SOA) interaction in Experiment 2. $\mathrm{S}_{1}$ (the first stimulus) modality was informative ( $80 \%$ validity for congruent trials). $\mathrm{RT}=$ response time; $\mathrm{VV}=$ visual $S_{1}$ followed by a visual $S_{2}$ (subsequent stimulus); $A V=$ auditory $S_{1}$ followed by a visual $\mathrm{S}_{2}$; $\mathrm{AA}=$ auditory $\mathrm{S}_{1}$ followed by an auditory $\mathrm{S}_{2}$; $\mathrm{VA}=$ visual $\mathrm{S}_{1}$ followed by an auditory $\mathrm{S}_{2}$. validity ( $50 \%$ vs. $80 \%$ ), and the within-participants factor was type of trial (ipsimodal vs. cross-modal). There was a significant Validity $\times$ Type of Trial interaction, $F(1,47)=26.672, p<.001$. Planned comparisons showed that responses were not significantly faster on ipsimodal trials in Experiment 2 than in Experiment 1 (mean difference $=20 \mathrm{~ms}, n s$ ), whereas they were slower on cross-modal trials in Experiment 2 than in Experiment 1 (mean difference $=65 \mathrm{~ms}, p<.01)$.

\section{Discussion}

The results confirmed that participants were able to use information provided by $S_{1}$ to attend to the cued modality, showing that the endogenous allocation of attention to the congruent-cued modality produced shorter RTs on valid trials than on invalid trials. Because of the presence of an endogenous component, we expected that the difference between valid-congruent and invalidincongruent trials should have been greater in Experiment 2 than in Experiment 1 . That is, we predicted shorter RTs on ipsimodal trials in the $80 \%$-validity than in the $50 \%$-validity condition and longer RTs on cross-modal trials in the $80 \%$-validity than in the $50 \%$ validity condition. As expected, at the shortest SOA (150 ms; i.e., the only SOA at which the advantage was present in both experiments), RTs on cross-modal trials were longer in Experiment 2 than in Experiment 1.

By contrast, although the effect was in the predicted direction, the advantage of ipsimodal trials over cross-modal trials was not significant. This might have been so because the task was very easy (simple detection), and therefore the endogenous component did not produce robust additional benefits in comparison with the exogenous one. A further signature for the endogenous component in the present study is that RTs on ipsimodal trials were shorter than RTs on cross-modal trials at both the 600- and 1,000-ms SOAs. The advantage of valid-congruent trials over invalidincongruent trials depended on SOA, being the greatest at the shortest SOA and the smallest at the 1,000-ms SOA. This, from our standpoint, occurs because in Experiment 2, both the automatic and the voluntary components acted together. Although we interpret the present findings as due to attention, one might observe that, because of the $80 \%$ validity of the cue, and because the task required a simple detection, the occurrence of a criterion shift cannot be ruled out, because the rate of false alarms was too small to detect differences between conditions.

\section{Experiment 3}

The results of Experiments 1 and 2 suggest that attention shifts between visual and auditory modalities are governed by exogenous and endogenous mechanisms. In particular, from Experiment 2, we know that a person can direct attention to a given modality using an informative $S_{1}$. Experiment 3 explored whether attention can be allocated to a cued modality even when $S_{1}$ predicts an incongruent $\mathrm{S}_{2}$ on $80 \%$ of trials (also see Posner et al., 1976).

With this experimental manipulation, the exogenous and the endogenous components should act, at least for the shortest SOA, in opposite directions. This is because, as soon as $S_{1}$ is perceived, attention is automatically summoned to its modality. However, participants are required to direct attention to the opposite modality. So, at the $150-\mathrm{ms} \mathrm{SOA}$, the advantage of valid trials over invalid trials should be reduced. By contrast, at the longest SOAs 
(600 and 1,000 ms), the exogenous component should be no longer effective, allowing the endogenous component to act in isolation. Therefore, when the $S_{1}-S_{2}$ interval is long enough, we should observe short RTs on valid cross-modal trials and long RTs on invalid ipsimodal trials.

\section{Method}

Participants. Twenty-five psychology students of the University of Padua (11 female and 14 male) participated. They were unaware of the purpose of the experiment and had not taken part in the previous experiments. Their mean age was 25 years (range $=22-27$ years), and they were selected as in previous experiments.

Apparatus and materials. The apparatus and materials were the same as in the previous experiments.

Design and procedure. The design and procedure were the same as in Experiment 2, except that valid trials ( $80 \%$ probability) were cross-modal, VA and AV, and invalid trials ( $20 \%$ probability) were ipsimodal, VV and AA. As before, there were three experimental blocks of 60 trials each (plus catch trials). There were 20 trials for each SOA, 16 trials for the incongruent modality $\mathrm{S}_{1}-\mathrm{S}_{2}$ combination ( $8 \mathrm{VA}$ and $8 \mathrm{AV}$ ), and 4 trials for the congruent modality $\mathrm{S}_{1}-\mathrm{S}_{2}$ combination ( $2 \mathrm{AA}$ and $2 \mathrm{VV}$ ). Catch trials were 12 in each block (about $17 \%$ of total trials).

\section{Results}

Overall false alarms were very rare, 3 out of 450 total responses for auditory $S_{1}$ and 5 out of 450 for visual $S_{1}$, and were not further analyzed. The outliers-latency criterion removed less than $3 \%$ of the total trials. RTs were entered into a repeated measures ANOVA in which the factors were those already reported in Experiments 1 and 2 . Both the main effect of $\mathrm{S}_{1}$ modality, $F(1$, $24)=9.258, p<.005$, and the main effect of $S_{2}$ modality, $F(1$, $24)=37.330, p<.001$, were significant. RTs were shorter for the auditory $\mathrm{S}_{1}(M=428 \mathrm{~ms}, S D=89)$ than for the visual $\mathrm{S}_{1}(M=$ $439 \mathrm{~ms}, S D=95)$, and shorter for the auditory $\mathrm{S}_{2}(M=418 \mathrm{~ms}$, $S D=87)$ than for the visual $\mathrm{S}_{2}(M=450 \mathrm{~ms}, S D=94)$. The main effect of SOA was significant, $F(2,48)=37.604, p<.001$, with shorter RTs at the two longer SOAs (150-ms SOA, $M=463 \mathrm{~ms}$, $S D=85 ; 600-\mathrm{ms} \mathrm{SOA}, M=401 \mathrm{~ms}, S D=82 ; 1,000$-ms SOA, $M=437 \mathrm{~ms}, S D=98)$. A significant $\mathrm{S}_{1}$ Modality $\times \mathrm{S}_{2}$ Modality interaction was found, $F(1,24)=49.924, p<.001(\mathrm{VV}, M=480$ $\mathrm{ms}, S D=84$; AV, $M=420 \mathrm{~ms}, S D=94$; AA, $M=437 \mathrm{~ms}, S D=$ 84; VA, $M=398 \mathrm{~ms}, S D=91)$, with shorter RTs on valid (incongruent) trials $(M=409 \mathrm{~ms}, S D=89)$ than on invalid (congruent) trials $(M=459 \mathrm{~ms}, S D=80)$. The $\mathrm{S}_{1}$ Modality $\times \mathrm{S}_{2}$ Modality $\times$ SOA interaction was also significant, $F(2,48)=$ $61.512, p<.001$, showing that the difference between valid trials and invalid trials varied as a function of SOA (see Figure 4 and Table 1).

Planned comparisons were applied to the data. At the 150-ms SOA, RTs in VV did not differ from those in AV. By contrast, AA showed shorter RTs than VA (mean difference $=28 \mathrm{~ms}, p<.01$ ). At the 600-ms SOA, RTs in VV were longer than in AV (mean difference $=75 \mathrm{~ms}, p<.01$ ), and RTs in AA were longer than in VA (mean difference $=62 \mathrm{~ms}, p<.01$ ). Similarly, at the 1,000-ms SOA, VV produced longer RTs than AV did (mean difference $=98 \mathrm{~ms}, p<.01$ ), and AA produced longer RTs than VA did (mean difference $=84 \mathrm{~ms}, p<.01$ ).

Because in the present experiment we expected that at the shortest SOA $(150 \mathrm{~ms})$ the advantage of valid trials over invalid

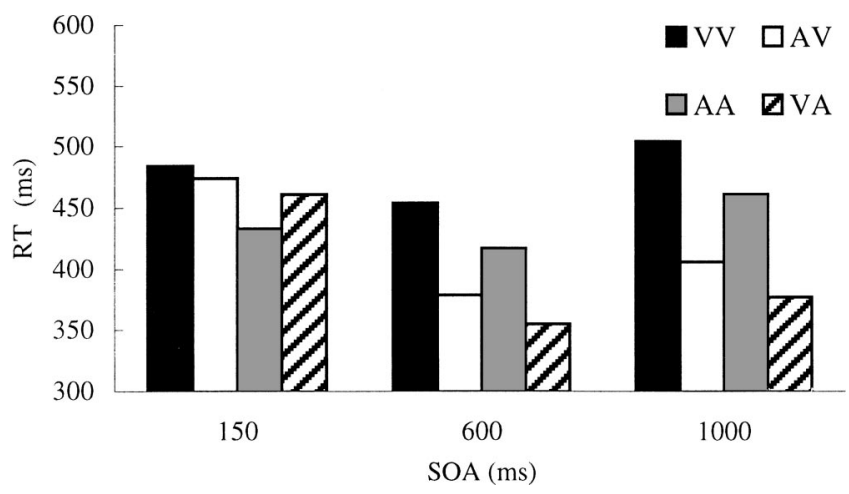

Figure 4. The $\mathrm{S}_{1}$ Modality $\times \mathrm{S}_{2}$ Modality $\times$ Stimulus Onset Asynchrony (SOA) interaction in Experiment 3. $\mathrm{S}_{1}$ (the first stimulus) modality was informative ( $80 \%$ validity for incongruent trials). $\mathrm{RT}=$ response time; $\mathrm{VV}=$ visual $\mathrm{S}_{1}$ followed by a visual $\mathrm{S}_{2}$ (subsequent stimulus); $\mathrm{AV}=$ auditory $S_{1}$ followed by a visual $S_{2}$; $A A=$ auditory $S_{1}$ followed by an auditory $S_{2}$; VA $=$ visual $S_{1}$ followed by an auditory $S_{2}$.

trials would be reduced in comparison with Experiment 2, we compared the validity effect (i.e., the difference between invalid and valid RTs) in the two experiments. The validity effect was greater in Experiment 2 than in Experiment 3, for both vision (136 ms vs. $10 \mathrm{~ms}, p<.01)$ and audition $(212 \mathrm{~ms}$ vs. $-28 \mathrm{~ms}, p<.01)$.

\section{Discussion}

The first conclusion we can draw from the results of the present experiment is that participants were able to direct attention to a given modality using a stimulus previously presented in a different modality. These results confirm those of Posner et al. (1976, Experiment 3), who showed that a visual informative $S_{1}$ was successful in facilitating detection of a subsequent auditory $S_{2}$.

It is worth noting that, when the interval between $S_{1}$ and $S_{2}$ was brief, and participants were presented with a visual $S_{1}$, they were unable to endogenously shift attention to audition quickly. Very often, in studies investigating cross-modal spatial links between vision and audition, a visual $S_{1}$ is used to indicate either a visual or an auditory $S_{2}$ (e.g., Spence \& Driver, 1997b). On the basis of the present findings, it would seem that this is not a safe procedure. In fact, when the SOA is short, the visual cue is more effective for a visual than for an auditory target. In accordance with what was noted in the discussion of Experiment 2, the present results might be interpreted on the basis of a criterion shift.

\section{Experiment 4}

The present experiment further explored the exogenous mechanism, trying to assess its degree of automaticity. Because the most demanding criterion for assessing automaticity is intentionality (Jonides, 1981), we used it to test the hypothesis that the occurrence of a stimulus produces a shift of attention to its sensory modality. To this purpose, in the present experiment, participants were informed that $\mathrm{S}_{2}$ modality remained fixed within a block of trials (100\% valid trials), whereas $S_{1}$ could have been either visual or auditory. This way, participants could allocate attention to $S_{2}$ modality in advance, trying to ignore, if possible, $\mathrm{S}_{1}$ modality. 


\section{Method}

Participants. Twenty-four psychology students of the University of Padua (12 female and 12 male) participated. They were unaware of the purpose of the experiment and had not taken part in any of the previous experiments. Their mean age was 23 years (range $=21-25$ years), and they were selected as in previous experiments.

Apparatus and materials. The apparatus and materials were as in the previous experiments.

Design and procedure. The design and procedure were as in Experiment 2, except that participants knew in advance that $S_{2}$ modality was fixed within a block of trials. $S_{1}$ modality was either congruent $(50 \%)$ or incongruent (50\%). Thus, regardless of $\mathrm{S}_{1}$ modality, in the visual condition $\mathrm{S}_{2}$ was always a visual stimulus, whereas in the auditory condition $\mathrm{S}_{2}$ was always an auditory stimulus. Order of presentation for the visual and auditory conditions was counterbalanced across participants.

There were two experimental blocks of 90 trials each (plus catch trials). There were 30 trials for each SOA, 15 trials for the congruent modality cue-target combination, and 15 for the incongruent modality cue-target combination. Catch trials were 16 in each block (about $15 \%$ of total trials). There were 30 trials for each SOA, 15 trials for the incongruent modality $\mathrm{S}_{1}-\mathrm{S}_{2}$ combination (VA or AV), and 15 trials for the congruent modality $\mathrm{S}_{1}-\mathrm{S}_{2}$ combination (AA or VV).

\section{Results}

Overall false alarms were rare, 6 out of 384 total responses for auditory $S_{1}$ and 1 out of 384 for visual $S_{1}$, and were not further analyzed. As a result of the outliers-latency criterion, less than $1 \%$ of trials were trimmed. RTs were entered into a repeated measures ANOVA in which the factors were SOA (150, 600, and 1,000 ms), modality of $S_{1}$ (visual and auditory), and modality of $S_{2}$ (visual and auditory). The main effect of $\mathrm{S}_{1}$ modality was significant, $F(1$, $23)=19.434, p<.001$, with participants responding more rapidly after an auditory $(M=373 \mathrm{~ms}, S D=81)$ than a visual $\mathrm{S}_{1}(M=$ $390 \mathrm{~ms}, S D=97)$. The main effect of $S_{2}$ modality was not significant, $F(1,23)<1$. Like in every previous experiment, the main effect of SOA was significant, $F(2,46)=57.827, p<.001$, with shorter RTs at the two longer SOAs (150-ms SOA, $M=428$ $\mathrm{ms}, S D=94 ; 600-\mathrm{ms}$ SOA, $M=349 \mathrm{~ms}, S D=66$; $1,000-\mathrm{ms}$ SOA, $M=368 \mathrm{~ms}, S D=87$ ).

A significant $\mathrm{S}_{1}$ Modality $\times \mathrm{S}_{2}$ Modality interaction was found, $F(1,23)=28.772, p<.001(\mathrm{VV}, M=376 \mathrm{~ms}, S D=75 ; \mathrm{AV}$, $M=384 \mathrm{~ms}, S D=79$; AA, $M=362 \mathrm{~ms}, S D=82$; VA, $M=404$ $\mathrm{ms}, S D=114)$, with shorter RTs for congruent than incongruent trials for an auditory $S_{2}$ and a similar, although weaker, trend for a visual $S_{2}$. The $S_{1}$ Modality $\times S_{2}$ Modality $\times$ SOA interaction was also significant, $F(2,46)=16.120, p<.001$ (see Figure 5).

Planned comparisons were applied to the data. At the 150-ms SOA, RTs in VV did not differ from those in AV. By contrast, AA showed shorter RTs than VA (mean difference $=101 \mathrm{~ms}, p<$ .01 ). At the 600- and 1,000-ms SOAs, no significant differences were found between $\mathrm{VV}$ and $\mathrm{AV}$ and between $\mathrm{AA}$ and VA.

\section{Discussion}

On the basis of Experiment 1, we concluded that the mere presentation of a stimulus tends to capture attention to the corresponding modality. However, an involuntary attentional capture can be said to occur only when participants are given the opportunity to fully focus attention to a given source of stimulation. Hence, in the present experiment, participants were clearly informed that, within a block of trial, $\mathrm{S}_{2}$ modality was fixed.

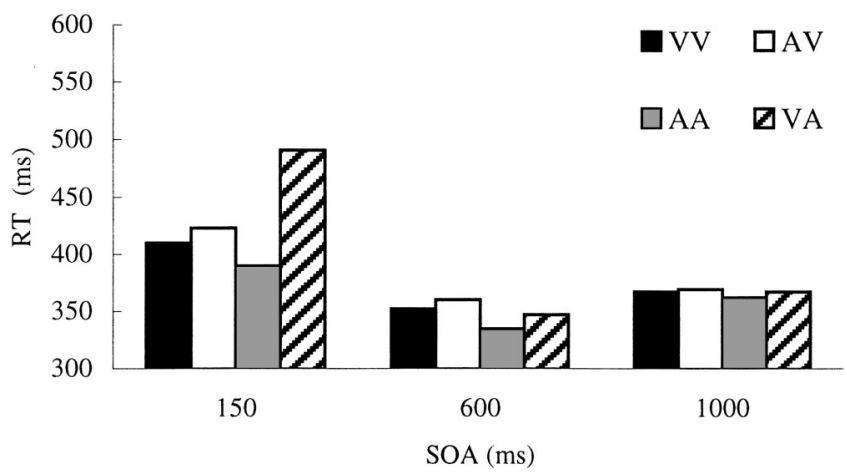

Figure 5. The $\mathrm{S}_{1}$ Modality $\times \mathrm{S}_{2}$ Modality $\times$ Stimulus Onset Asynchrony (SOA) interaction in Experiment $4 . \mathrm{S}_{2}$ (subsequent stimulus) modality was always $100 \%$ valid, whereas $S_{1}$ (the first stimulus) modality was either congruent $(50 \%)$ or incongruent $(50 \%)$. $\mathrm{RT}=$ response time; $\mathrm{VV}=$ visual $S_{1}$ followed by a visual $S_{2} ; A V=$ auditory $S_{1}$ followed by a visual $S_{2}$; $\mathrm{AA}=$ auditory $\mathrm{S}_{1}$ followed by an auditory $\mathrm{S}_{2}$; VA $=$ visual $\mathrm{S}_{1}$ followed by an auditory $S_{2}$.

The pattern of results may reflect an asymmetry between modalities in automatically summoning attention (but see Experiment 5). Regardless of SOA, when $S_{2}$ was a visual stimulus, $S_{1}$ modality did not affect performance. By contrast, when $S_{2}$ was an auditory stimulus, and SOA was short, a visual $\mathrm{S}_{1}$ produced longer RTs in comparison with an auditory $\mathrm{S}_{1}$. At longer SOAs (600 and 1,000 $\mathrm{ms}), \mathrm{S}_{1}$ modality did not matter. The present findings suggest that, when attention is fully allocated to vision, detection of a visual $S_{2}$ is not affected by an auditory $S_{1}$. In contrast, when attention is fully allocated to audition, detection of an auditory $S_{2}$ is slowed down by a visual $S_{1}$. Alternatively one might observe that, at the 150-ms SOA, RTs in AA were particularly long because, when unattended, a visual $S_{1}$ is less alerting than an auditory $S_{1}$ (also see Posner et al., 1976).

Such asymmetry might also occur because stimuli presented in the two modalities were not of the same intensity, with visual stimuli being stronger than auditory stimuli. However, this explanation is ruled out by two observations. First, at the 1,000-ms SOA, no RT difference emerged between vision and audition, which suggests that visual stimuli were not more intense than auditory stimuli. Second, at the 150-ms SOA, RTs on ipsimodal trials were shorter for audition than for vision (also see Experiment 1). Hence, a difference in stimulus intensity, if present, would have predicted an auditory dominance rather than a visual one.

A further aspect of the present findings is hard to reconcile with a criterion shift account. In fact, it is difficult to see how a criterion shift might have affected the results of Experiment 4, in which an incongruent visual $S_{1}$ lengthened RTs to the auditory $S_{2}$, whereas an incongruent auditory $S_{1}$ did not lengthen RTs for the visual $S_{2}$. Hence, unless one assumes that in the present experiment a criterion shift occurred only in one modality, a criterion-shift account is not tenable (see Ward, McDonald, \& Lin, 2000). However, to provide even stronger evidence that the present results were not affected by a criterion shift, we performed Experiment 5, in which a discrimination task was used.

\section{Experiment 5}

Experiment 5 was identical to Experiment 4, except that participants performed a choice RT task instead of a simple RT task. A 
discrimination task should prevent participants from lowering their criterion for responding before $S_{2}$ is presented. In the case of a visual $S_{2}$, participants had to discriminate between the LED flashing in red or in green color. In the case of an auditory $S_{2}$, participants were required to discriminate between a low-pitch and a high-pitch tone. One may observe that the tasks for the two modalities were different, and therefore a task shift (e.g., Rogers \& Monsell, 1995) might have occurred on cross-modal trials. However, the occurrence of a task shift would seem unlikely, considering that $\mathrm{S}_{2}$ modality was blocked, and therefore participants had no uncertainty about the task they had to perform on $\mathrm{S}_{2}$.

\section{Method}

Participants. Thirty-two psychology students of the University of Padua (23 female and 9 male) participated. They were unaware of the purpose of the experiment and had not taken part in any of the previous experiments. Their mean age was 24.1 years (range $=20-31$ years), and they were selected as in the previous experiments.

Apparatus and materials. The apparatus and materials were as in the previous experiments.

Design and procedure. The design and procedure were as in Experiment 4 , with the following exceptions. For the visual modality, $S_{1}$ was the onset of the LED in orange, whereas for the auditory modality, $\mathrm{S}_{1}$ was a $900+1800-\mathrm{Hz}$ tone. The visual $\mathrm{S}_{2}$ consisted of the LED appearing in red or green, whereas the auditory $\mathrm{S}_{2}$ consisted of a low-pitch tone $(900 \mathrm{~Hz})$ or high-pitch tone $(1800 \mathrm{~Hz})$. Participants had to react as quickly as possible by pressing the $H$ key when $\mathrm{S}_{2}$ was the green light or the low-pitch tone and the $B$ key when $\mathrm{S}_{2}$ was the red light or the high-pitch tone.

The total number of trials was 192, divided into four experimental blocks of 48 trials each. Specifically, there were 16 trials for each SOA, 8 trials for the congruent modality $S_{1}-S_{2}$ combination, and 8 for the incongruent modality $\mathrm{S}_{1}-\mathrm{S}_{2}$ combination.

\section{Results}

$R T$ analysis. As a result of the outliers-latency criterion, less than $2 \%$ of trials were trimmed. RT data were entered into a repeated measures ANOVA in which the factors were those already reported in all previous experiments. The main effects of $S_{1}$ modality and $\mathrm{S}_{2}$ modality were not significant $(F<1)$.

The main effect of SOA was significant, $F(2,62)=136.498, p$ $<.001$, with shorter RTs at the two longer SOAs (150-ms SOA, $M=610 \mathrm{~ms}, S D=104 ; 600-\mathrm{ms}$ SOA, $M=510 \mathrm{~ms}, S D=83$; 1,000 -ms SOA, $M=488 \mathrm{~ms}, S D=88$ ). Only the $\mathrm{S}_{1}$ Modality $\times$ $\mathrm{S}_{2}$ Modality $\times$ SOA interaction was significant, $F(2,62)=$ 16.159, $p<.001$ (see Figure 6).

Planned comparisons were applied to the data. At the 150-ms SOA, for either modality, ipsimodal trials were faster than crossmodal trials (auditory $\mathrm{S}_{2}$, mean difference $=34 \mathrm{~ms}, p<.01$; visual $\mathrm{S}_{2}$, mean difference $=30 \mathrm{~ms}, p<.05$ ). At the 600 -ms SOA, ipsimodal trials did not differ from cross-modal trials. At the 1,000-ms SOA, for either modality, ipsimodal trials were slower than cross-modal trials (auditory $\mathrm{S}_{2}$, mean difference $=43 \mathrm{~ms}$, $p<.001$; visual $\mathrm{S}_{2}$, mean difference $=18 \mathrm{~ms}, p<.05$ ).

Error analysis. Overall error rate was less than $4 \%$. An ANOVA with the usual factors did not reveal any statistical differences in error distribution across conditions, with the secondorder interaction resulting in nonsignificance $(F<1)$.

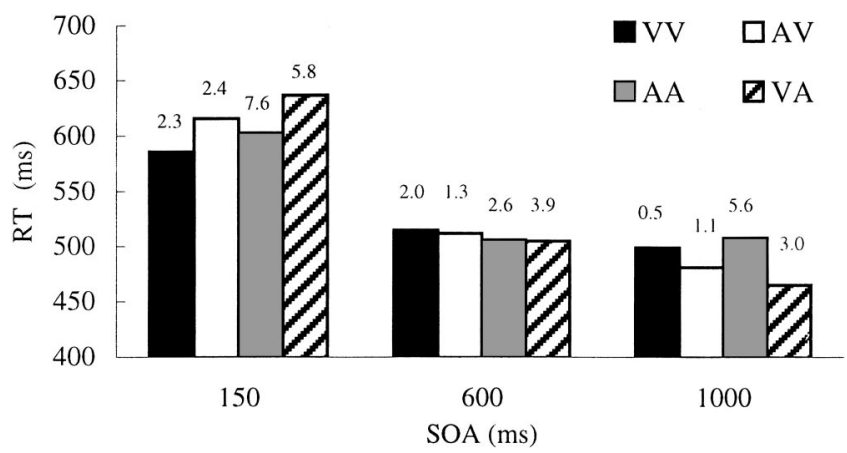

Figure 6. The $\mathrm{S}_{1}$ Modality $\times \mathrm{S}_{2}$ Modality $\times$ Stimulus Onset Asynchrony (SOA) interaction in Experiment 5. $\mathrm{S}_{2}$ (subsequent stimulus) modality was always $100 \%$ valid, whereas $S_{1}$ (the first stimulus) modality was either congruent $(50 \%)$ or incongruent $(50 \%)$. Task consisted of a discrimination of either the light-emitting diode color or the pitch tone. Numbers above the bars indicate percentage error rates. $\mathrm{RT}=$ response time; $\mathrm{VV}=$ visual $S_{1}$ followed by a visual $S_{2}$; AV $=$ auditory $S_{1}$ followed by a visual $S_{2}$; $\mathrm{AA}=$ auditory $\mathrm{S}_{1}$ followed by an auditory $\mathrm{S}_{2} ; \mathrm{VA}=$ visual $\mathrm{S}_{1}$ followed by an auditory $S_{2}$.

\section{Discussion}

The main result of the present experiment was that, for either modality, an exogenous attentional capture emerged at the shortest SOA. These findings, obtained with a choice RT task, confirmed and extended those from Experiments 1 and 4. Most important, the attentional capture observed was obligatory, in that it took place despite participants knowing in advance $S_{2}$ modality, thus having the opportunity to fully allocate attention to that modality. Because we used a discrimination task, any version of a criterion shift account can be definitely ruled out.

Unlike Experiment 4, in the present experiment, a cross-modal advantage was evident at the longest SOA. An explanation of such an effect might be that, because $S_{2}$ was certain, participants tried to inhibit $S_{1}$, whose processing was not only useless but could have been somewhat detrimental for performing the task. This potentially distracting effect is indeed attested by the obligatory attentional capture elicited by cross-modal trials at the shortest SOA. If the SOA was long $(1,000 \mathrm{~ms})$, then participants had enough time to endogenously inhibit $S_{1}$. Because $S_{2}$ modality was blocked, the effect of this inhibitory mechanism of $S_{1}$ modality was evident only on ipsimodal trials. Perhaps the cross-modal advantage at the longest SOA was not evident in Experiment 4 because with an easier task (simple detection) inhibiting $S_{1}$ was not necessary.

Another difference between Experiments 4 and 5 is that, in the present experiment, no asymmetry in attentional capture between modalities emerged at the shortest SOA. As Ward, McDonald, and Golestani (1998) have argued for reconciling Ward's (1994) and Spence and Driver's (1997a) data on exogenous cross-modal shifts of spatial attention, perhaps subtle changes in experimental conditions and in task demands play an important role in producing different patterns of results (an asymmetry in Experiment 4; no asymmetry in Experiment 5). The difference between Experiments 4 and 5 at the shortest SOA could also occur because, in Experiment 4 (simple RT), following an auditory $S_{1}$ participants became slightly less conservative, thus producing shorter RTs in AA and $\mathrm{AV}$ than in VV and VA. 


\section{Experiment 6}

Because it was not clear whether a criterion shift might have played a role in Experiment 1, we deemed it important to further investigate whether attention may be exogenously attracted by an uninformative $S_{1}$ when participants have to perform a discrimination task on $S_{2}$. On the basis of the results of Experiment 5, we expected to replicate the exogenous effect when $S_{1}-S_{2}$ interval was very brief, thus also replicating the findings of Experiment 1 for the shortest SOA. We did not make any prediction for the longest SOA, although, in principle, we might have expected the automatic attentional capture to dissipate with comparatively long SOAs.

\section{Method}

Participants. Twenty-eight psychology students of the University of Padua (26 female and 2 male) participated. They were unaware of the purpose of the experiment and had not taken part in any of the previous experiments. Their mean age was 24.2 years (range $=19-27$ years), and they were selected as in the previous experiments.

Apparatus and materials. The apparatus and materials were the same as in Experiment 5.

Design and procedure. The design and procedure were as in Experiment 5, with the exception that $S_{2}$ modality was not blocked but rather was unpredictable and varied from trial to trial within a block. The total number of trials was 192, divided into four experimental blocks of 48 trials each. There were 16 trials for each SOA, 8 trials for the congruent modality $\mathrm{S}_{1}-\mathrm{S}_{2}$ combination (4 VV and $4 \mathrm{AA}$ ), and 8 for the incongruent modality $\mathrm{S}_{1}-\mathrm{S}_{2}$ combination (4 AV and $4 \mathrm{VA}$ ).

\section{Results}

$R T$ analysis. As a result of the outliers-latency criterion, about $1 \%$ of trials were trimmed. RT data were entered into a repeated measures ANOVA in which the factors were those already reported in all previous experiments. The main effect of $S_{1}$ modality was significant, $F(1,27)=4.813, p<.04$, with participants responding overall faster after an auditory $\mathrm{S}_{1}(M=690 \mathrm{~ms}, S D=$ $89)$ than after a visual $\mathrm{S}_{1}(M=705 \mathrm{~ms}, S D=85)$. The main effect of $\mathrm{S}_{2}$ modality was also significant, $F(1,27)=8.294, p<.01$, with participants responding overall faster to a visual $S_{2}(M=674$ $\mathrm{ms}, S D=89)$ than to an auditory $\mathrm{S}_{2}(M=721 \mathrm{~ms}, S D=101)$. The main effect of SOA was significant, $F(2,54)=92.845, p<.001$, with shorter RTs at the two longer SOAs (150-ms SOA, $M=755$ $\mathrm{ms}, S D=97 ; 600-\mathrm{ms}$ SOA, $M=668 \mathrm{~ms}, S D=79$; $1,000-\mathrm{ms}$ SOA, $M=670 \mathrm{~ms}, S D=83)$. The $\mathrm{S}_{1}$ Modality $\times \mathrm{S}_{2}$ Modality interaction was significant, $F(1,27)=29.509, p<.001$, as well as the $\mathrm{S}_{2}$ Modality $\times$ SOA interaction, $F(2,54)=17.278, p<$ .001 . More important, the second-order $S_{1}$ Modality $\times S_{2}$ Modality $\times$ SOA interaction was also significant, $F(2,54)=3.983, p<$ .03 (see Figure 7).

Planned comparisons were applied to the data. At the 150-ms SOA, for both modalities, ipsimodal trials were faster than crossmodal trials (auditory $\mathrm{S}_{2}$, mean difference $=86 \mathrm{~ms}, p<.002$; visual $\mathrm{S}_{2}$, mean difference $=50 \mathrm{~ms}, p<.001$ ). At the $600-\mathrm{ms}$ SOA, ipsimodal trials were still faster than cross-modal trials (auditory $\mathrm{S}_{2}$, mean difference $=63 \mathrm{~ms}, p<.001$; visual $\mathrm{S}_{2}$, mean difference $=33 \mathrm{~ms}, p<.006$ ). At the $1,000-\mathrm{ms} \mathrm{SOA}$, for the auditory modality only, ipsimodal trials were faster than crossmodal trials (mean difference $=36 \mathrm{~ms}, p<.004$ ).

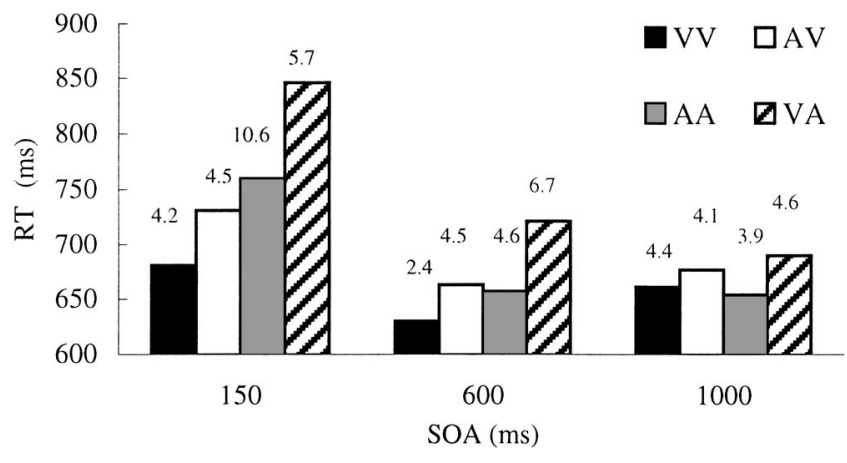

Figure 7. The $\mathrm{S}_{1}$ Modality $\times \mathrm{S}_{2}$ Modality $\times$ Stimulus Onset Asynchrony (SOA) interaction in Experiment 6. $\mathrm{S}_{1}$ (the first stimulus) modality was not informative of $\mathrm{S}_{2}$ (subsequent stimulus) modality. Task consisted of a discrimination of either the light-emitting diode color or the pitch tone. Numbers above the bars indicate percentage error rates. $\mathrm{RT}=$ response time; $\mathrm{VV}=$ visual $\mathrm{S}_{1}$ followed by a visual $\mathrm{S}_{2} ; \mathrm{AV}=$ auditory $\mathrm{S}_{1}$ followed by a visual $S_{2}$; AA auditory $S_{1}$ followed by an auditory $S_{2}$; VA = visual $S_{1}$ followed by an auditory $S_{2}$.

Error analysis. Overall error rate was less than 6\%. An ANOVA with the usual factors did not reveal any statistical differences in errors distribution across conditions, with the second-order interaction resulting in nonsignificance $(F<1)$.

\section{Discussion}

The main purpose of the present experiment was to replicate the automatic attentional capture that had emerged at the shortest SOA in both Experiment 1 and Experiment 5. Results confirmed that at 150-ms SOA ipsimodal trials were faster than cross-modal trials.

In our view, the exogenous ipsimodal facilitation is evidence that, once $S_{1}$ is perceived, attention is automatically grabbed by the corresponding modality, facilitating $\mathrm{S}_{2}$ discrimination when it is in the same modality as compared with when it is in a different modality. Because $S_{1}$ was not predictive of $S_{2}$ modality, we might have expected, for the longer SOAs (600 and 1,000 ms), the automatic ipsimodal facilitation to be no longer present. Instead, this ipsimodal advantage was still present at both intervals. Because the task was harder than the simple RT task of Experiment 1 , and because on $50 \%$ of trials the subsequent $S_{2}$ was ipsimodal, it is plausible that once attention was grabbed by a modality, participants maintained attention in that modality.

Taken together, the results of the six experiments presented showed that an auditory $S_{1}$ did not facilitate the processing of a visual $S_{2}$ more than a visual $S_{1}$ did. By contrast, early studies (e.g., Harvey, 1980; Posner et al., 1976) had suggested that RTs to a visual target were shorter after an auditory warning signal than after a visual warning signal. There might be several reasons for this discrepancy. For instance, consider Experiment 2 of Posner et al., which has the same rationale and design as our Experiment 6 . Apart from the fact that Posner et al. did not provide any physical measure of stimuli intensity, which renders a direct comparison difficult, their conclusion that an auditory warning signal $\left(\mathrm{S}_{1}\right)$ was more alerting than a visual signal appears to be too strong. In fact, such cross-modal facilitation occurred only at the shortest $S_{1}-S_{2}$ interval. In addition, at this interval errors were more numerous in the cross-modal (13) than in the ipsimodal condition (7), which might index a speed-accuracy trade-off. 
The notion that auditory stimuli are more alerting than visual ones for responding to a visual $\mathrm{S}_{2}$ was also supported by Harvey (1980). However, his results are somewhat unclear. In Experiment 1, Harvey used stimuli that were comparable in terms of intensity to those we have used here, and he found that $\mathrm{AV}$ were faster than $\mathrm{VV}$ in all the interstimulus intervals (ISIs) of 50, 350, and $550 \mathrm{~ms}$. However, it should be noted that, when data are collapsed across $S_{1}$ and $S_{2}$ category, at the shortest ISI, VA were faster than AA, a result that is clearly incompatible with the notion that auditory stimuli are more alerting than visual ones.

In addition, there is an aspect of the results of both Posner et al. (1976) and Harvey (1980) that renders it difficult to interpret $S_{1}$ as a warning signal. That is, in Posner et al.'s Experiment 2, RT increased as a function of SOA, whereas in Harvey's cross-modal condition, RT was not affected by ISI. In contrast, RT should decrease as the interval between the warning signal and the imperative stimulus increases (e.g., Bertelson, 1967, and the six experiments of the present study), at least up to about 1,000 ms.

Finally, it may be worth noting that in either the study of Posner et al. (1976) or of Harvey (1980), not only were visual and auditory sources of stimulation spatially separated, but they were also located in different sectors of spaces. Visual stimuli, provided on the computer screen, were located in peripersonal space, if not in extrapersonal space. Auditory stimuli, delivered throughout headphones, were located in personal space. There is evidence showing that personal (near) space and peripersonal or extrapersonal (far) space are coded and represented separately in the human brain (e.g., Berti \& Frassinetti, 2000; Umilta, 2000a). By contrast, in all the experiments reported in the present study, stimuli from visual and auditory modalities originated from peripersonal space. At the moment it is difficult to figure out how this difference may have affected the results. However, it might well be that the alerting effect of an auditory stimulus varies as a function of whether it falls in different sectors of spaces.

\section{General Discussion}

The purpose of the present study was to investigate exogenous and endogenous attentional shifts between vision and audition in the absence of any spatial components. Previous studies had already shown that when two stimuli are presented one after the other, the modality of $\mathrm{S}_{1}$ affects the processing of $\mathrm{S}_{2}$ (e.g., Harvey, 1980; Ponser et al., 1976). An asymmetric effect between auditory and visual modalities was reported, with an auditory $S_{1}$ facilitating the discrimination of a visual $S_{2}$. Such asymmetry was hypothesized to be in relation with both the low alerting capability of visual stimuli and the automatic alerting effect of auditory stimuli. In particular, it was suggested that an auditory $S_{1}$ would be able to activate central processing mechanism(s) without the intervention of attention, thus rendering decision mechanisms ready to process inputs from any modalities (Posner et al., 1976).

Our hypothesis (also see the MSE; Spence \& Driver, 1997b), instead, predicted that attention is required to consciously process any sensory input, and therefore we expected $S_{1}$ to facilitate the detection or discrimination of an ipsimodal $\mathrm{S}_{2}$ in comparison with a cross-modal $S_{2}$. That would be because in the former case attention is already allocated in the modality of the target, whereas in the latter case it needs to be shifted between the two modalities before $\mathrm{S}_{2}$ discrimination and/or response selection might take place.
The experimental conditions of the present study were planned to avoid the methodological artifacts (criterion shift, response priming, and spatial-cuing effect) that rendered equivocal the results of previous studies (Spence \& Driver, 1997b). Participants performed either a simple or a choice RT task, in which $\mathrm{S}_{2}$ modality was suggested by $S_{1}$ modality with a trial-by-trial procedure. Under these conditions, it can be assumed that response priming had no role, and especially so in the case of the choice RT task. In addition, and very importantly, the sources of stimulation for audition and vision were spatially coincident, eliminating any spatial-cuing component. In theory, a criterion shift might have been present in Experiments 1 to 4, which were based on a simple detection task. However, the analysis of accuracy on catch trials showed that the percentage of false alarms was negligible in all the experiments. That shows that participants followed the instructions very carefully and did not change their criterion for responding. At any rate, results from Experiments 5 and 6, which were based on a discrimination task, basically confirmed those obtained with the simple detection task. That rules out a criterion-shift explanation.

A first conclusion that can be drawn on the basis of the present results is that observers are able to use an informative $S_{1}$ to allocate attention endogenously to the modality of $S_{2}$, when $S_{2}$ is either ipsimodal (Experiment 2) or cross-modal (Experiment 3). Note that in the cross-modal condition participants had to direct attention to vision if a sound was perceived, and vice versa. On the basis of the findings of Experiments 1 and 2, we expected that, at the shortest SOA, the endogenous mechanism would be in conflict with the exogenous mechanism triggered by an incongruent $S_{1}$, thus decreasing the advantage for valid cross-modal trials over invalid ipsimodal trials. By contrast, for the longer SOAs (600 and $1,000 \mathrm{~ms}$ ), after the exogenous component dissipated, the endogenous mechanism should direct attention efficiently. The results confirmed these predictions, showing that when the $S_{1}-S_{2}$ delay was very short $(150 \mathrm{~ms})$, the endogenous mechanism was probably counteracted by the exogenous mechanism, whereas for longer SOAs, participants were able to shift attention between modalities also using an $\mathrm{S}_{1}$ incongruent with $\mathrm{S}_{2}$ modality.

In our view, the exogenous attentional capture observed when the $S_{1}-S_{2}$ interval was very brief is of particular interest. It shows that when a visual or an auditory $S_{1}$ is provided, attention is briefly grabbed by the corresponding modality (Experiments 1 and 6). Depending on whether $S_{2}$ is delivered in the same or in a different modality, two results can be observed: a short-lived attentional facilitation when the two stimuli are ipsimodal, and a short-lived attentional limitation when the two stimuli are cross-modal. This purely exogenous effect, which seems to be mandatory for vision and audition (Experiments 4 and 5), dissipates rapidly over time, being present only in the first $200 \mathrm{~ms}$ from $\mathrm{S}_{1}$ presentation.

Once it is established that the presentation of a visual or auditory stimulus produces an automatic attentional capture to the corresponding modality, an interesting issue is to evaluate the degree of automaticity of such capture. Using a discrimination task, Experiment 5 clearly showed that the onset of either a visual or an auditory $S_{1}$ produced a mandatory allocation of attention to the stimulated sensory channel. However, it should be mentioned that, with a simple RT task (Experiment 4), we found such obligatory capture to be asymmetric for vision and audition. In fact, when attention was fully allocated to vision, a sudden onset of an auditory $S_{1}$ did not produce an obligatory shift of attention to audition, whereas when attention was fully allocated to audition, 
an abrupt visual onset captured attention to vision. As of now, we do not have an explanation for why the mandatory capture of attention between modalities should be asymmetric with a simple detection task and symmetric with a discrimination task.

The fact that, at the shortest SOA, for either vision or audition, ipsimodal trials were faster than cross-modal trials casts doubt on the arguments proposed by Posner et al. (1976; also see Klein, 1977 ) to explain why the visual dominance phenomenon occurs in experiments with RTs. In fact, we did not find evidence either that visual stimuli were less alerting than auditory stimuli or that the latter exhibited an automatic alerting effect. Hence, the present data do not confirm the notion that the visual modality would be more poorly linked to alerting mechanisms than the auditory modality. The only instance in which we found an asymmetric effect of visual and auditory stimuli was with a simple RT task in Experiment 4 . However, data showed that an auditory $\mathrm{S}_{1}$ was not able to grab attention when attention was fully allocated to vision, whereas a visual $S_{1}$ grabbed attention in a stimulus-driven manner when participants were fully focused on audition. This might suggest that, in the present task, visual inputs tend to dominate over auditory inputs, and that visual dominance, if any, would not occur because attention is biased to vision because visual inputs have a less efficient priority interrupt than audition. Rather, on the basis of Experiment 4, visual dominance might be conceived as a high priority interrupt for visual inputs, which causes an obligatory processing of visual stimuli. Further research is needed to clarify why this sign of visual dominance emerged with a simple RT task but not with a discrimination task.

The short-latency cross-modal attentional interference between visual and auditory stimuli we documented is also of relevance for another reason. A growing bulk of evidence in the literature indicates that, when two target stimuli $\mathrm{T}_{1}$ and $\mathrm{T}_{2}$, each one requiring a response ( Task $_{1}$ and $\mathrm{Task}_{2}$ ), are presented in close temporal proximity, interference in processing $\mathrm{T}_{2}$ is usually found (e.g., Pashler, 1994). This would happen because performance on Task postpones the availability of attention to $\mathrm{Task}_{2}$, or, more generally, central mechanisms, which operate serially, are not available for processing $\mathrm{T}_{2}$ if they are still engaged in the processing of $\mathrm{T}_{1}$ (Pashler, 1994; Van Selst \& Jolicoeur, 1997). Two welldocumented examples of dual-task interference are the psychological refractory period (PRP; e.g., Pashler, 1994) and the attentional blink phenomenon (AB; e.g., Raymond, Shapiro, \& Arnell, 1992). Although traditionally the central-processing limitation issue has been investigated by presenting $T_{1}$ and $T_{2}$ in the same modality, recently it has been shown that such limitation also occurs when $\mathrm{T}_{1}$ and $\mathrm{T}_{2}$ are in different modalities (e.g., Arnell \& Jolicoeur, 1999; De Jong, 1993; Dell'Acqua, Turatto, \& Jolicoeur, 2001). These findings seem to suggest that the central mechanisms responsible for the attentional bottleneck in the flow of information processing are modality independent.

It is interesting to note that, here, we have reported a delay in processing $S_{2}$ (or $T_{2}$ ) when a cross-modal $S_{1}$ was presented $150 \mathrm{~ms}$ in advance, participants not having to perform any task on $S_{1}$. Why has this cross-modal limitation emerged? Dual-task interference in both PRP and $\mathrm{AB}$ is thought to occur because stages of stimulus categorization and/or response selection cannot be shared between tasks, which thus must be performed serially (Pashler, 1994). However, in our Experiments 5 and 6, in which exogenous crossmodal interference was evident, participants were neither required to categorize $S_{1}$ nor required to select any response to it. Hence, it appears that the between-modalities attentional limitation we have documented cannot be accounted for by these central processing mechanisms. Instead we hypothesize that once $S_{1}$ is presented, regardless of whether it has to be actively processed or ignored, central mechanisms are briefly automatically set to the corresponding modality, facilitating the processing of an ipsimodal $\mathrm{S}_{2}\left(\right.$ or $\mathrm{T}_{2}$ ) but delaying attentional resources for central processing of a cross-modal $\mathrm{S}_{2}$.

Dell'Acqua et al. (2001) reported a series of experiments in which $\mathrm{T}_{1}$ and $\mathrm{T}_{2}$ were delivered to two distinct modalities at various SOAs, with each stimulus associated with a different task ( Task ${ }_{1}$ and Task $_{2}$ ). In their Experiments 2 to 4, they showed that when participants attended to $\mathrm{T}_{1}$ modality, a performance decrement of $\mathrm{T}_{2}$ discrimination occurred, providing evidence for a cross-modal AB. Accuracy on Task $_{2}$ decreased when the $T_{1}-T_{2}$ delay was brief, being the lowest for the two shortest SOAs (100 and $250 \mathrm{~ms}$ ), and recovering completely at the 900-ms SOA. Such an SOA-locked impairment was nearly entirely eliminated when $\mathrm{T}_{1}$ could be ignored. However, it is worth noting that even when participants were required to ignore $T_{1}$, in all the experiments, accuracy on $\mathrm{T}_{2}$ was lower at the two shortest SOAs than at the longest SOA (5\% on average). As suggested, perhaps the mere presentation of information in a to-be-ignored $\mathrm{T}_{1}$ modality captures attention to the corresponding sensory channel, or, in general, central mechanisms are temporarily and exogenously set to process information from the activated modality. This would cause a brief shortage of attentional resources for processing a $T_{2}$ in a different modality. Such a modality-dependent mechanism might be responsible for the modest, although evident, performance decrement in Task 2 in Dell'Acqua et al.'s study, even when participants did not have to perform Task ${ }_{1}$.

Although we have provided evidence for a cross-modal attentional limitation, many studies have generated consistent results showing a cross-modal attentional facilitation (see Driver \& Spence, 1998, for a review). How can these apparently discrepant results be reconciled? We believe the discrepancy can be accommodated in light of the distinction between two types of attention, input attention and central attention, proposed by Johnston, McCann, and Remington (1995). The two types of attention have been proposed to affect in different manners the process of stimulus selection. Whereas the former has the function of selecting stimulus location, the latter would be involved in making complex decisions about stimulus properties. If one considers our study, in which both vision and audition shared the same spatial source, it is clear that we were mainly concerned with central attention. In these conditions, we have shown that central mechanisms produce a brief cross-modal attentional limitation. By contrast, studies on cross-modal spatial attention deal with input attention and show that a spatially uninformative $S_{1}$ in one modality grabs attention to its location also in other modalities, thus exhibiting a cross-modal facilitatory effect for $\mathrm{S}_{2}$. Cross-modal facilitation or limitation would then be caused by different attentional mechanisms, and the experimental conditions one adopts would cause the former or the latter to emerge.

\section{References}

Arnell, K. M., \& Jolicoeur, P. (1999). The attentional blink across stimulus modalities: Evidence for central processing limitations. Journal of Experimental Psychology: Human Perception and Performance, 25, 630648. 
Bertelson, P. (1967). The time course of preparation. Quarterly Journal of Experimental Psychology: Human Experimental Psychology, 19(A), 272-279.

Berti, A., \& Frassinetti, F. (2000). When far becomes near: Remapping of space by tool use. Journal of Cognitive Neuroscience, 12, 415-420.

Boulter, L. R. (1977). Attention and reaction times to signals of uncertain modality. Journal of Experimental Psychology: Human Perception and Performance, 3, 379-388.

Colavita, F. B. (1974). Human sensory dominance. Perception \& Psychophysics, 16, 409-412.

De Jong, R. (1993). Multiple bottlenecks in overlapping task performance. Journal of Experimental Psychology: Human Perception and Performance, 19, 965-989.

Dell'Acqua, R., Turatto, M., \& Jolicoeur, P. (2001). Cross-modal attentional deficits in processing tactile stimulation. Perception \& Psychophysics, 63, 777-789.

Driver, J., \& Spence, C. (1994). Spatial synergies between auditory and visual attention. In C. Umiltà \& M. Moscovitch (Eds.), Attention and performance XV: Conscious and nonconscious information processing (pp. 311-331). Cambridge, MA: MIT Press.

Driver, J., \& Spence, C. (1998). Attention and the crossmodal construction of space. Trends in Cognitive Sciences, 7, 254-262.

Egeth, H. E., \& Sager, L. C. (1977). On the locus of the visual dominance. Perception \& Psychophysics, 22, 77-86.

Gibson, J. J. (1943). Adaptation, after-effect, and contrast in the perception of curved lines. Journal of Experimental Psychology, 16, 1-31.

Harvey, N. (1980). Non-informative effects of stimuli functioning as cues. Quarterly Journal of Experimental Psychology: Human Experimental Psychology, 32(A), 413-425.

Johnston, J. C., McCann, R. S., \& Remington, R. W. (1995). Chronometric evidence for two types of attention. Psychological Science, 6, 365-369.

Jonides, J. (1981). Voluntary versus automatic control over the mind's eye's movement. In J. B. Long \& A. D. Baddeley (Eds.), Attention and performance IX (pp. 187-203). Hillsdale, NJ: Erlbaum.

Klein, R. M. (1977). Attention and visual dominance: A chronometric analysis. Journal of Experimental Psychology: Human Perception and Performance, 3, 365-378.

Klein, R. M. (2000). Inhibition of return. Trends in Cognitive Sciences, 4, $138-147$.

Kristofferson, M. W. (1967). Shifting attention between modalities. Journal of Abnormal Psychology, 72, 388-394.

Moore, J. J., \& Massaro, D. W. (1973). Attention and processing capacity in auditory recognition. Journal of Experimental Psychology, 99, 49-54.

Nickerson, R. S. (1973). Intersensory facilitation of reaction time. Psychological Review, 80, 489-509.

Pashler, H. (1994). Dual-task interference in simple tasks: Data and theory. Psychological Bulletin, 116, 19-45.

Posner, M. I., \& Cohen, Y. (1984). Components of visual orienting. In H. Bouma \& D. G. Bouwhuis (Eds.), Attention and performance $X$ (pp. 531-556). Hillsdale, NJ: Erlbaum.

Posner, M. I., Nissen, M. J., \& Klein, R. M. (1976). Visual dominance: An information-processing account of its origins and significance. Psychological Review, 83, 157-171.

Raymond, J. E., Shapiro, K. L., \& Arnell, K. M. (1992). Temporary suppression of visual processing in RSVP task: An attentional blink? Journal of Experimental Psychology: Human Perception and Performance, 18, 849-860.

Rogers, R. D., \& Monsell, S. (1995). Costs of predictable switch between simple cognitive tasks. Journal of Experimental Psychology: General, 124, 207-231

Sanders, A. F. (1975). The foreperiod effect revisited. Quarterly Journal of Experimental Psychology: Human Experimental Psychology, 27(A), 591-598.

Schneider, W. (1988). Micro Experimental Laboratory: An integrated system for IBM-PC compatibles. Behavior Research Methods, Instruments, and Computers, 20, 206-217.

Shiffrin, R. M., \& Grantham, D. W. (1974). Can attention be allocated to sensory modalities? Perception \& Psychophysics, 15, 460-474.

Spence, C., \& Driver, J. (1994). Covert spatial orienting in audition: Exogenous and endogenous mechanisms. Journal of Experimental Psychology: Human Perception and Performance, 20, 555-574.

Spence, C., \& Driver, J. (1996). Audiovisual links in endogenous covert spatial attention. Journal of Experimental Psychology: Human Perception and Performance, 22, 1005-1030.

Spence, C., \& Driver, J. (1997a). Audiovisual links in exogenous covert spatial orienting. Perception \& Psychophysics, 59, 1-22.

Spence, C., \& Driver, J. (1997b). On measuring selective attention to an expected sensory modality. Perception \& Psychophysics, 59, 384-403.

Umiltà, C. (2000a). Mechanisms of attention. In B. Rapp (Ed.), The handbook of cognitive neuropsychology (pp. 135-158). New York: Psychology Press.

Umiltà, C. (2000b). Visuospatial attention. In F. Boller \& J. Grafman (Eds.), Handbook of neuropsychology (2nd ed., pp. 393-425). Amsterdam: Elsevier.

Van Selst, M., \& Jolicoeur, P. (1997). Decision and response in dual-task interference. Cognitive Psychology, 33, 266-307.

Verleger, R., \& Cohen, R. (1978). Effects of certainty, modality shift and guess outcome on evoked potentials and reaction times in chronic schizophrenics. Psychological Medicine, 8, 81-93.

Ward, L. M. (1994). Supramodal and modality-specific mechanisms for stimulus-driven shifts of auditory and visual attention. Canadian Journal of Experimental Psychology, 48, 242-259.

Ward, L. M., McDonald, J. J., \& Golestani, N. (1998). Cross-modal control of attention shifts. In R. D. Wright (Ed.), Visual attention (pp. 232-268). New York: Oxford University Press.

Ward, L. M., McDonald, J. J., \& Lin, D. (2000). On asymmetries in cross-modal spatial attention orienting. Perception \& Psychophysics, 62, $1258-1264$.

Received August 26, 1999

Revision received August 9, 2001

Accepted September 11, 2001 